\title{
EDITORIAL
}

\section{Themenschwerpunkt „Logistik“}

\author{
Andreas D. Lattner · Ingo J. Timm
}

Online publiziert: 22. Mai 2010

(C) Springer-Verlag 2010

\section{Liebe Leserinnen, liebe Leser,}

Logistik umfasst verschiedene Aufgaben, welche die Planung und Steuerung von Prozessen zum Transport von Entitäten bspw. Güter und Daten betreffen. Hierbei sind Problemstellungen auf unterschiedlichen Ebenen vom Management bis hin zum Materialfluss zu lösen. Durch die hohe Komplexität der logistischen Szenarien und Entscheidungsprozesse sowie eine mögliche Verteilung auf verschiedene Organisationen ist die Unterstützung durch KITechnologien notwendig oder zumindest für die Optimierung von Abläufen zur Einsparung von Zeit und Kosten hilfreich. Wie die Beiträge in diesem Schwerpunktheft zeigen, bestehen hierbei viele Möglichkeiten, Methoden aus der Künstlichen Intelligenz einzusetzen. Der Fachbeitrag von Günther und Nissen betrachtet die untertägige Personaleinsatzplanung für einen Logistikdienstleister. Die Problemstellung wird in dem Beitrag durch Particle Swarm Optimization und Evolutionsstrategien angegangen. Die Behandlung dynamischer Ereignisse wird in dem Fachbeitrag von Sauer sowie dem Projektbeitrag von Pulter, Nimis und Lockemann adressiert. Im ersten Fall wird die vertikale Datenintegration für ein reaktives Scheduling in Supply Chains diskutiert. Pulter et al. präsentieren das Projekt LogoTakt, in dem ein Störungsmanagement für offene, getaktete Logistiknetze entwickelt wird. Hellingrath und Böhle diskutieren in ihrem Fachbeitrag den Einsatz von Multiagentensystemen für die Produktions-und Logistikplanung in der Supply Chain, wobei insbesondere diskutiert wird, wie die Entwicklung von Agentensystemen erfolgen könnte, um die Anfor-

A.D. Lattner $(\bowtie) \cdot$ I.J. Timm

Wirtschaftsinformatik und Simulation, Goethe-Universität Frankfurt (FB 12), Postfach 111932, 60054 Frankfurt am Main, Deutschland

e-mail: lattner@informatik.uni-frankfurt.de derungen des Supply Chain Managements besser berücksichtigen. Der Beitrag von Gehrke, Herzog, Langer, Malaka, Porzel und Warden gibt einen Überblick über den agentenbasierten Ansatz im Sonderforschungsbereich 637 (,Selbststeuerung logistischer Prozesse - Ein Paradigmenwechsel und seine Grenzen“). Zwei weitere Projektbeiträge präsentieren die Anwendung von KI-Methoden in der Logistik. Smirnov und Shilov präsentieren einen Ansatz zur Lösung von dynamischen Transportproblemen u.a. unter der Verwendung eines Constraint-Solvers. Der Beitrag von Runkler, Grothmann und Bamberger beschreibt die Optimierung industrieller Logistikprozesse mit Schwarmintelligenz und rekurrenten neuronalen Netzen. Hierbei wird der Einsatz an verschiedenen Projekten illustriert: Preis-und Absatzprognosen, Fertigungslogistik und Flotten-Management. In der Dissertation von Pawlaszczyk wird ein Ansatz zur skalierbaren, agentenbasierten Simulation vorgestellt, was für komplexe Szenarien wie in der Transportlogistik von Bedeutung ist. Die Dissertation von Döring behandelt den Einsatz von Reinforcement Learning zur Steuerung der Änderungsplanung in Produktionsnetzwerken der Serienfertigung. Abgerundet wird dieses Schwerpunktheft durch ein Interview mit Paul Davidsson, dem Leiter des Distributed and Intelligent Systems Laboratory (DISL) am Blekinge Institute of Technology in Schweden - einem Logistikexperten, der insbesondere auch Agenten in der Sozialsimulation eingesetzt hat. Wir bedanken uns bei den Autoren für die interessanten Beiträge und bei den KI-Editoren für die Gelegenheit zur Gestaltung dieses Schwerpunkthefts sowie für die Unterstützung bei der Erstellung. Wir wünschen Ihnen viel Freude beim Lesen und hoffen, dass laufende oder auch neue Forschungsvorhaben durch die Beiträge in diesem Heft inspiriert werden!

Andreas D. Lattner und Ingo J. Timm 